\title{
Electrical and Mechanical Activities Recorded from Smooth Muscle Cells of the Human Fallopian Tube
}

\author{
Tadao Kishikawa and Hiroshi Kuriyama \\ Department of Pharmacology, Faculty of Medicine, \\ Kyushu University, Fukuoka, 812 Japan
}

\begin{abstract}
Summary The membrane activity recorded from the circular muscle layer of the human Fallopian tube showed a unique pattern (slow potential changes), yet the hormonal and adrenergic influences appeared to be much the same as observed in the myometrium. Differences in electrical and mechanical activities, and also the sensitivity to catecholamines during the secretory or proliferative period were elucidated.
\end{abstract}

The role of the human Fallopian tube during the reproduction process has long been discussed in relation to the ovum transportation from the measurement of contraction and from volume changes in vitro and in vivo (CoutinHo et al., 1973; Hodgson et al., 1977; Croxatto et al., 1978; Pauerstein and EdDy, 1979). Recently, KORENAGA (1980) measured the contraction of circular rings, in vitro, of the human Fallopian tube under careful measurements of background conditions, namely contents of progesterone and estradiol in sera of patients with myoma in relation to the stage of ovarian cycle, and changes in the basal body temperature until the date of hysterectomy. He concluded that in the absence of domination of ovarian steroid hormones, adrenoceptors in the circular muscle of human Fallopian tube seem to be $\beta$-dominant. Estradiol increases the sensitivity of $\alpha$-adrenoceptors thereby contributing to regulation of the mechanical activity in the amplitude and frequency. In the secretory phase, progesterone enhances the $\beta$-adrenoceptors, thus producing contractions of irregular amplitude.

In general, the mechanical activity is triggered by a change in the membrane property, namely spike or slow potential changes as observed in various visceral smooth muscles. However, these cells were surrounded by thick elastic and connective tissues, therefore, the electrical activity of smooth muscles of the human Fallopian tube has not been recorded due to technical difficulties.

To understand the mechanism involved in ovum transport, electrical and mechanical activities of the smooth muscle cells of Fallopian tube were recorded, and the results were discussed in relation to hormonal dominations.

Received for publication October 23, 1980

岸川忠雄, 栗山 熙 
In the Japanese patients (54 patients; $21-52$ years old), the unilateral Fallopian tube was excised following a diagnosis of myoma in the Kyushu University Hospital. The stage of the ovarian cycle at the date of the operation was recorded. Immediately after excision, the tissue was placed in oxygenated Krebs solution $\left(12-16^{\circ} \mathrm{C}\right)$, and brought to our laboratory. The procedures conformed to the Declaration of Helsinki.

To record the electrical activity using the microelectrode method, the tissue was prepared in $3 \mathrm{~mm}$ width and $10 \mathrm{~mm}$ length, and for the double sucrose gap method, $1 \mathrm{~mm}$ width and $10 \mathrm{~mm}$ length. Using a binocular microscope, the muscle strips were mainly excised from the circular muscle layer of the ampullar and isthmus regions. The glass capillary electrode filled with $3 \mathrm{M} \mathrm{KCl}$ was used, and the resistance of the electrode ranged between 50 and $80 \mathrm{M} \Omega$. The experimental procedures were the same as those described in the case of the rat myometrium by KANDA and KURIYAMA (1980). The double sucrose gap method was adapted by the method used for the vascular smooth muscle by ITo et al. (1977).

The solution used in the present experiments was a modified Krebs solution introduced by BüLBRING and KURIYAMA (1963), and the $\mathrm{pH}$ of the solution was kept at 7.2-7.3 by oxygenation with $97 \% \mathrm{O}_{2}$ and $3 \% \mathrm{CO}_{2}$.

The membrane potential of circular muscle cells of the ampullar region of Fallopian tube was $-50.9 \pm 3.1 \mathrm{mV}$ S.D. $(n=65)$ during the secretory period and this value was much the same as that measured during the proliferative period $(-52.1 \pm 1.9 \mathrm{mV}$ S.D.; $n=58)$. No regional difference between the ampullar and isthmus regions was apparent at any stage of the ovarian cycle. When the microelectrode was inserted into the circular muscle of the ampullar region in the Fallopian tube excised from a patient during menopause, the membrane potential was $-46.9 \pm 4.7 \mathrm{mV}$ S.D. ( 2 patients; 49 and 52 years old).

The spontaneous membrane activity recorded from the muscle cell in all regions of the Fallopian tube showed a slow potential change (slow wave). The amplitude and frequency of these slow changes varied from cell to cell $(15-41 \mathrm{mV}$ and $1.0-0.02 \mathrm{~Hz}$, respectively) under different hormonal conditions. The amplitude was less dependent, but the frequency and regularity of the amplitude depnded on hormonal domination, i.e., the frequency was lower and the amplitude was irregular in the progesterone-dominated condition (excised during the early secretory period) compared to findings in the estrogen-dominated condition (excised during the proliferative period).

Figure 1 shows the relationship between the membrane and mechanical activities recorded from the ampullar region of different preparations at various hormonal stages. Regular amplitude of the slow potential change with relatively low frequency was recorded from the cell during the secretory period. The mechanical activity, in contrast to the electrical activity, was irregular and not synchronized with the electrical activity (a). This phenomenon indicates that irregular amplitudes of contractions observed during the progesterone-dominated condition are 

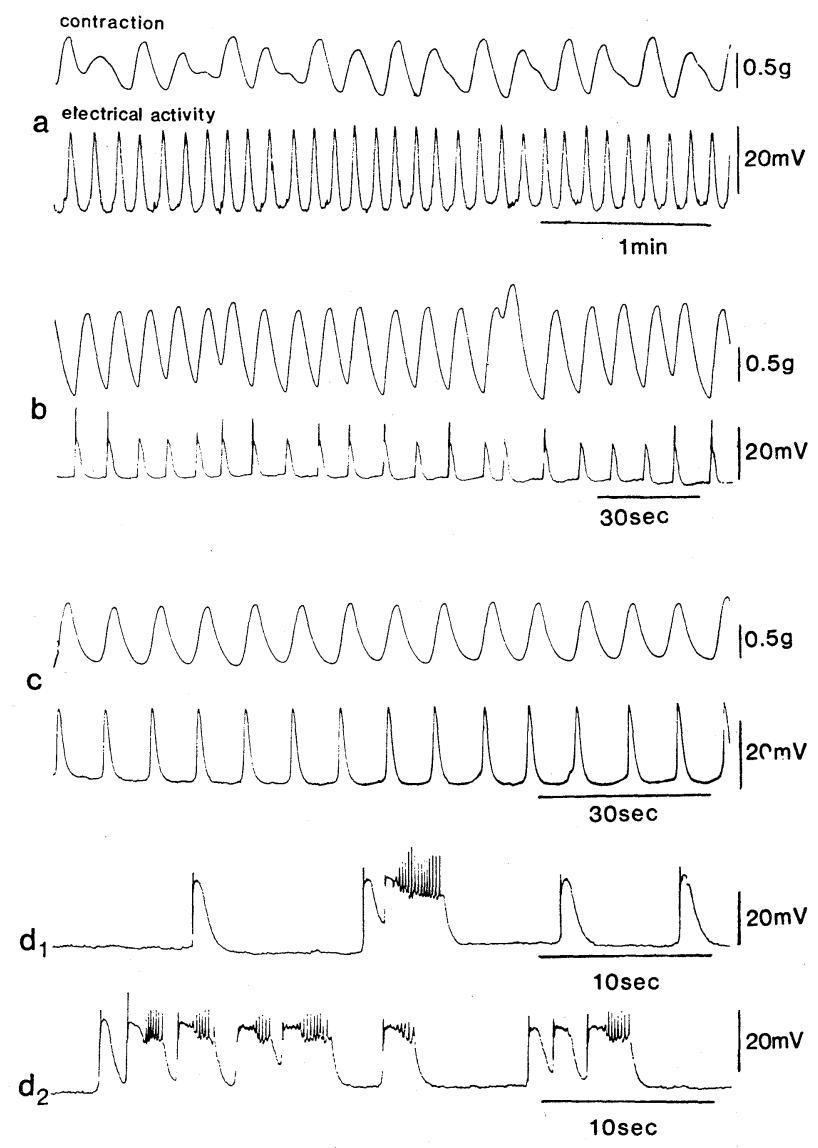

Fig. 1. Electrical and mechanical activities recorded from circular muscle cells of the ampullar region of the Fallopian tube. Contractions were recorded from the whole tissue and electrical activities were recorded from single cells using a microelectrode. $\mathrm{a}$, activities during the secretory period (progesterone-dominated condition); b, activities on the day of ovulation; $c$, activities during the proliferative period (estrogendominated condition); $d_{1}-d_{2}$, continuous records. Electrical activities during the proliferative period.

not due to an irregular generation of the slow potential change but rather to desynchronization of cellular activities in the tissue. In $b$, the electrical and mechanical activities recorded from the circular muscle of the ampullar region at the day of ovulation are shown. In some cells, the spike was superimposed on the slow potential change. The electrical activity was well synchronized with mechanical activity. Figure 1c shows the electrical and mechanical activities recorded from the circular muscle of the ampullar region during the proliferative period. In contrast to the secretory or intermediate period, the electrical and mechanical activities were well synchronized. The amplitude of the slow potential change was 
more regular compared to findings during the secretory period. These observations might indicate that functional cell-to-cell connections are increased during the estrogen-dominated stage, such as has been found in the rat myometrium (KuriYAma and Suzuki, 1976).

Figure $1 d_{1}$ and $d_{2}$ show two different patterns of electrical activity recorded from the circular muscle of ampullar region during the proliferative period. There were two distinct differences in the electrical activity, namely, a single spike superimposed on the slow potential change and repetitive spikes superimposed on the plateau potential. These two electrical patterns had no causal relation regarding their appearance. The former showed a typical pattern of electrical activity usually observed in all regions of the Fallopian tube, and the latter a typical pattern of the electrical activity as was observed in the rat and mouse myometria during the mid-pregnancy or spayed myometria under estradiol-treated conditions.

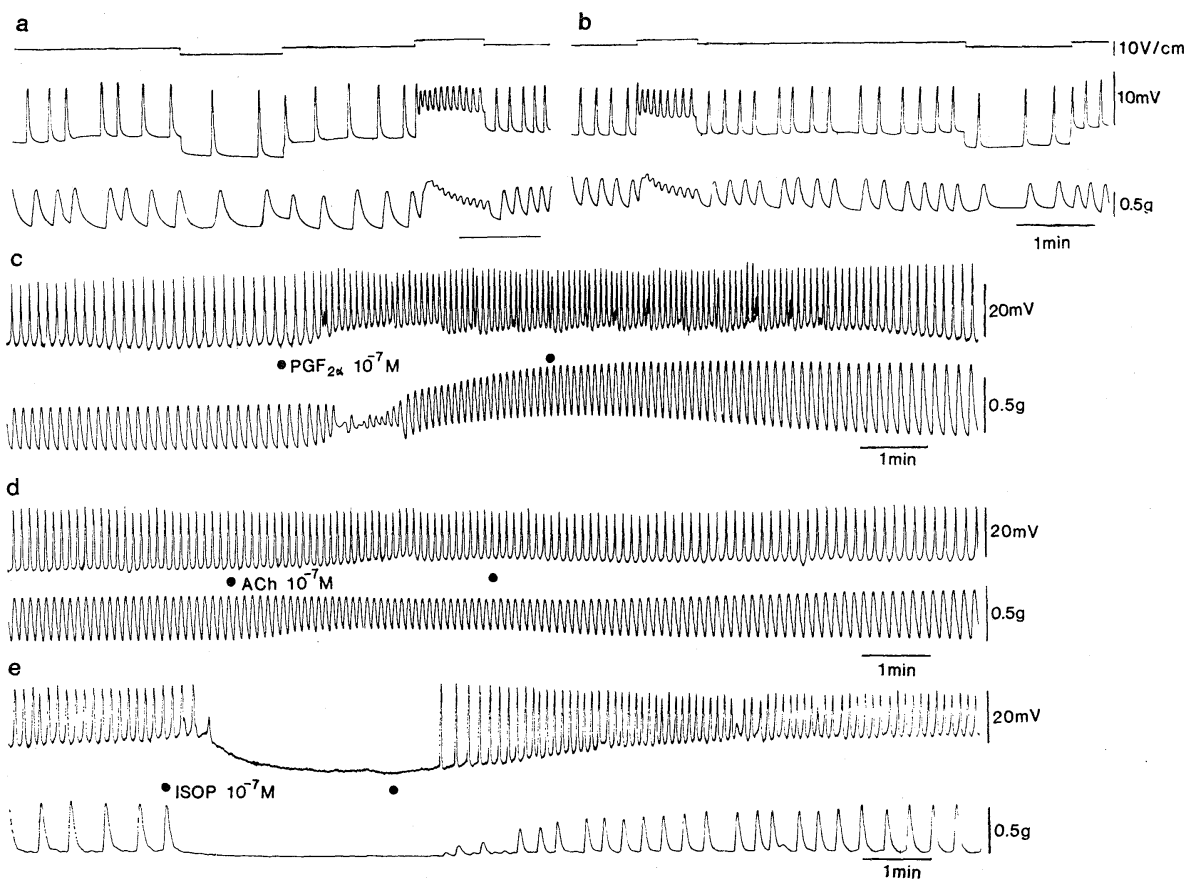

Fig. 2. a and b: Effects of electrical displacements of the membrane potential on the electrical and mechanical activities of circular muscle excised from the ampullar region of the Fallopian tube (proliferative period). In a and $b$, various intensities of inward and outward current pulses were applied. The double sucrose gap method was used. c-e: Effects of various chemical agents $\left(10^{-7} \mathrm{M}\right)$ on electrical and mechanical activities of single circular muscle cells of the ampullar region during the proliferative ( $c$ and $d$ ) and secretory phases (e). c, effects of prostaglandin $\mathrm{F}_{2 \alpha}\left(\mathrm{PGF}_{2 \alpha}\right)$; d, effects of acetylcholine ( $\left.\mathrm{ACh}\right)$; e, effects of isoprenaline (ISOP). Upper record, electrical activity; lower record, mechanical activity. The area between the dots indicates application of the chemical agent. 
The fundamental electrical activity recorded from the smooth muscle of the human Fallopian tube seems to be the generation of slow potential change, regardless of whether or not the spike is superimposed. These electrical activities resembled findings of the circular muscle of mammalian stomachs including that of humans (HARA and ITO, 1979). However, clear differences between the two tissues were apparent from the responses to electrical displacement of the membrane potential, i.e., in the human stomach, the frequency and amplitude of the slow wave were less dependent on the change in the membrane potential displaced by applications of inward and outward current pulses. On the other hand, the responses of the human Fallopian tube to electrical displacement of the membrane potential were remarkable. Figure $2 \mathrm{a}$ and $\mathrm{b}$ show the effects of electrical displacement of the membrane potential of the circular muscle of the human Fallopian tube excised from the ampullar region during the proliferative period. Hyperpolarization of the membrane increased the amplitude of the slow potential change and reduced the frequency. The reversed sequences were observed by depolarization of the membrane. These responses of the membrane were similar to those observed in the myometrium from pregnant rat (SUzUKI and KURIYAMA, 1975).

In the rat myometrium, responses of smooth muscle cells of longitudinal and circular muscles to catecholamines differed under different hormonal dominations (KAWARABAyashi and Osa, 1976; KaWARABAYASHI, 1978). Similar changes in the mechanical and electrical properties were also observed in smooth muscle cells of the Fallopian tube.

In the circular muscle of the ampulla, noradrenaline hyperpolarized the membrane and suppressed the electrical activity during the secretory period, while in the estradiol-dominated condition, noradrenaline produced a depolarization and accelerated the membrane activity. The former was suppressed by propranolol and the latter was suppressed by phentolamine. Furthermore, the action of isoprenaline on the circular muscle of ampullar region appeared more markedly during the progesterone-dominated condition (Fig. 2e) than during the estradioldominated condition. From the actions of catecholamines on the membrane activity, it is concluded that during the secretory period, $\beta$ - rather than $\alpha$-adrenoceptors seem to be more dominant in the circular muscle, while during the proliferative period, activations of $\alpha$-adrenoceptors become dominant. On the other hand, acetylcholine showed a weak excitatory action on the membrane (Fig. 2d), i.e., slightly depolarized and increased the frequency of the slow potential change regardless of the hormonal conditions. Muscarinic receptors seem to be sparsely distributed in the muscle cell of the human Fallopian tube. Actions of prostaglandin $\mathrm{F}_{2 \alpha}$ on electrical and mechanial activities as an excitatory substance also remained the same in different hormonal conditions (Fig. 2c).

Desynchronization or synchronization of electrical and mechanical activities and also differences in the sensitivity to catecholamine in circular muscle cells of the Fallopian tube during the secretory or proliferative period add to our knowl-

Vol. 31, No. 3, 1981 
edge of the physiological role of the Fallopian tube during the transportation of ovum to the uterus within one week after ovulation.

\section{REFERENCES}

BÜLBRING, E. and KURIYAMA, H. (1963) Effect of changes in external sodium and calcium concentration on spontaneous electrical activity in smooth muscle of guinea-pig taenia coli. $J$. Physiol. (Lond.), 166: 29-58.

Coutinho, E. M., Maria, H., and Costa, R. X. (1973) The effect of a continuous low dose progestin on tubal and uterine motility. Int. J. Fertil., 18: 161-166.

Croxatto, H. B., Ortiz, M. E., Diaz, S., Balmaceda, J., Hess, R., and Croxatto, H. D. (1978) Studies on the duration of egg transport by the human oviduct. Am. J. Obstet. Gynecol., 132: 629-634.

HARA, Y. and Ito, Y. (1979) The electrical activity recorded from smooth muscle of the circular layer of the human stomach. Pflügers Arch., 382: 145-153.

Hodgson, B. J., Talo, A., and Pauerstein, C. J. (1977) Oviductal ovum surrogate movement: Interrelation with muscular activity. Biol. Reprod., 16: 394-396.

Ito, Y., Suzuki, H., and KuRiYama, H. (1977) Effects of caffeine and procaine on the membrane and mechanical properties of the smooth muscle cells of the rabbit main pulmonary artery. Jpn. J. Physiol., 27: 467-481.

KANDA, S. and KurIYAMA, H. (1980) Specific feature of smooth muscle cells recorded from the placental region of the myometrium of pregnant rats. J. Physiol. (Lond.), 299: 127-144.

KAWARABAYASHI, T. (1978) The effects of phenylephrine in various ionic environments on the circular muscle of mid-pregnant rat myometrium. Jpn. J. Physiol., 28: 627-645.

KAWARABAYASH, T. and OsA, T. (1976) Comparative investigations of alpha- and beta-effects on the longitudinal and circular muscles of the pregnant rat myometrium. Jpn. J. Physiol., 26: 403-416.

KorenaGA, M. (1980) Functional change of the isthmic circular ring of the human oviduct. Bull. Res. Inst. Balneother., 31: 89-113 (in Japanese).

KuriYama, H. and Suzuki, H. (1976) Changes in electrical properties of rat myometrium during gestation and following hormonal treatments. J. Physiol. (Lond.), 260: 315-333.

Pauerstein, C. J. and EDdy, C. A. (1979) The role of the oviduct in reproduction: Our knowledge and our ignorance. J. Reprod. Fertil., 55: 223-229.

SuzuKI, H. and Kuriyama, H. (1975) Comparison between prostaglandin $E_{2}$ and oxytocin actions on the pregnant mouse myometrium. Jpn. J. Physiol., 25, 345-356.

Vol. 31, No. 3, 1981 